\title{
Keck/OSIRIS Pa $\beta$ High-contrast Imaging and Updated Constraints on PDS 70b
}

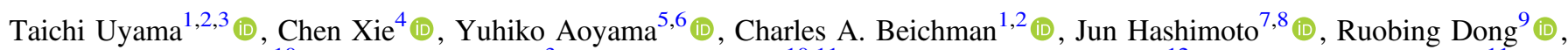

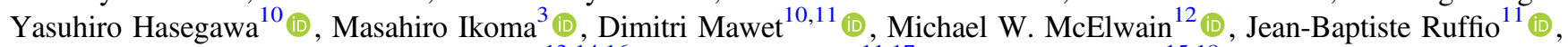 \\ Kevin R. Wagner ${ }^{13,14,16_{\text {DD }} \text {, Jason J. Wang }}{ }^{11,17}$ (D), and Yifan Zhou ${ }^{15,18}$ (D) \\ ${ }^{1}$ Infrared Processing and Analysis Center, California Institute of Technology, 1200 E. California Boulevard, Pasadena, CA 91125, USA; tuyama@ipac.caltech.edu \\ ${ }^{2}$ NASA Exoplanet Science Institute, Pasadena, CA 91125, USA \\ ${ }^{3}$ National Astronomical Observatory of Japan, 2-21-1 Osawa, Mitaka, Tokyo 181-8588, Japan \\ ${ }^{4}$ Aix Marseille Univ, CNRS, CNES, LAM, Marseille, France \\ ${ }^{5}$ Institute for Advanced Study, Tsinghua University, Beijing 100084, People's Republic of China \\ ${ }^{6}$ Department of Astronomy, Tsinghua University, Beijing 100084, People's Republic of China \\ Astrobiology Center, 2-21-1 Osawa, Mitaka, Tokyo 181-8588, Japan \\ ${ }^{8}$ Subaru Telescope, National Astronomical Observatory of Japan, Mitaka, Tokyo 181-8588, Japan \\ 9 Department of Physics \& Astronomy, University of Victoria, Victoria, BC V8P 1A1, Canada \\ ${ }^{10}$ Jet Propulsion Laboratory, California Institute of Technology, 4800 Oak Grove Drive, Pasadena, CA 91109, USA \\ ${ }_{11}^{11}$ Department of Astronomy, California Institute of Technology, 1200 E. California Boulevard, Pasadena, CA 91125, USA \\ ${ }^{12}$ Exoplanets and Stellar Astrophysics Laboratory, Code 667, Goddard Space Flight Center, Greenbelt, MD 20771, USA \\ ${ }^{13}$ Steward Observatory, University of Arizona, Tucson, AZ 85721, USA \\ ${ }^{14}$ NASA NExSS Alien Earths Team, USA \\ ${ }^{15}$ Department of Astronomy/McDonald Observatory, The University of Texas, 2515 Speedway, Stop C1400 Austin, TX 78712, USA \\ Received 2021 July 12; revised 2021 August 13; accepted 2021 September 14; published 2021 October 26
}

\begin{abstract}
We present a high-contrast imaging search for $\mathrm{Pa} \beta$ line emission from protoplanets in the PDS 70 system with Keck/OSIRIS integral field spectroscopy. We applied the high-resolution spectral differential imaging technique to the OSIRIS $J$-band data but did not detect the $\mathrm{Pa} \beta$ line at the level predicted using the parameters of Hashimoto et al. (2020). This lack of $\mathrm{Pa} \beta$ emission suggests the MUSE-based study may have overestimated the line width of $\mathrm{H} \alpha$. We compared our $\mathrm{Pa} \beta$ detection limits with the previous $\mathrm{H} \alpha$ flux and $\mathrm{H} \beta$ limits and estimated $A_{\mathrm{V}}$ to be $\sim 0.9$ and 2.0 for PDS $70 \mathrm{~b}$ and c, respectively. In particular, PDS $70 \mathrm{~b}$ 's $A_{\mathrm{V}}$ is much smaller than implied by highcontrast near-infrared studies, which suggests the infrared-continuum photosphere and the hydrogen-emitting regions exist at different heights above the forming planet.
\end{abstract}

Unified Astronomy Thesaurus concepts: Exoplanet astronomy (486); Planet formation (1241)

\section{Introduction}

A variety of theoretical and observational studies have investigated planet formation, yet the mechanisms are still poorly understood. High-contrast imaging at infrared (IR) wavelengths can detect the thermal emission of young exoplanets directly and thus provide key insights to distinguish between various planet formation mechanisms. Characterization of the physical and atmospheric parameters of protoplanets at specific ages helps in assessing the initial conditions of their formation (e.g., Bonnefoy et al. 2014). Furthermore, addressing such problems as reconciling the evolutionary cooling models (hot/warm/cold start; Spiegel \& Burrows 2012) with the relevant physical processes (e.g., core accretion and disk instability; Pollack et al. 1996; Boss 1997) are essential to improving our understanding of planet formation. One of the ways to probing planet formation is to observe hydrogen emission originating in active mass accretion onto protoplanets (Aoyama et al. 2018).

PDS 70 is one of the most intriguing young systems with highcontrast imaging, revealing two protoplanets located within a large cavity of the protoplanetary disk (PDS 70bc; Keppler et al. 2018; Haffert et al. 2019) and follow-up observations confirming active mass accretion onto them (e.g., Haffert et al. 2019). Previous studies have explored some of the hydrogen-emission lines

\footnotetext{
${ }^{16}$ NASA Hubble Fellowship Program-Sagan Fellow.

1751 Pegasi b Fellow.

${ }^{18}$ Harlan J. Smith McDonald Observatory Fellow.
}

in the PDS 70 system; $\mathrm{H} \alpha(656.28 \mathrm{~nm}), \mathrm{H} \beta(486.14 \mathrm{~nm}), \mathrm{Br} \alpha$ $(4.050 \mu \mathrm{m})$, and $\operatorname{Br} \gamma(2.166 \mu \mathrm{m})$. $\mathrm{H} \alpha$ emission has been reported by MagAO (Wagner et al. 2018), VLT/MUSE (Haffert et al. 2019), and HST (Zhou et al. 2021). The measured $\mathrm{H} \alpha$ flux shows temporal variability on a 1-2 yr timescale for reasons that are still controversial: either systematic instrumental calibration errors and/or an intrinsic time variability. The MUSE data include $\mathrm{H} \beta$ line but yielded only a null detection (Hashimoto et al. 2020) with $3 \sigma$ upper limits of 2.3 and $1.6 \times 10^{-16} \mathrm{erg} \mathrm{s}^{-1} \mathrm{~cm}^{-2}$ for PDS $70 \mathrm{~b}$ and c, respectively. Christiaens et al. (2019) reported the $K$-band spectrum of PDS $70 \mathrm{~b}$ taken by VLT/SINFONI $(R \sim 100)$ and Wang et al. (2021) presented the $K$-band spectra of PDS $70 \mathrm{bc}$ taken by VLT/GRAVITY (MEDIUM resolution), but they did not detect significant $\mathrm{Br} \gamma$ emission. Wang et al. (2021) set $3 \sigma$ upper limits of $\mathrm{Br} \gamma$ to 5.1 and $4.0 \times 10^{-17} \mathrm{erg} \mathrm{s}^{-1} \mathrm{~cm}^{-2}$ for PDS $70 \mathrm{~b}$ and c, respectively, which are limited by the $K$-band continua of PDS $70 \mathrm{bc}$. Stolker et al. (2020) reported the detection of PDS $70 \mathrm{bc}$ with VLT/NACO NB4.05 filter $(\mathrm{Br} \alpha$ filter; $\left.\lambda_{\text {cen }}=4.05 \mu \mathrm{m}, \Delta \lambda=0.02 \mu \mathrm{m}\right)$. However, they suggested that PDS 70 b's spectrum is best fit by an atmospheric model without $\operatorname{Br} \alpha$ and did not argue in favor of a line detection. In addition to the hydrogen-emission lines, Zhou et al. (2021) reported ultraviolet (UV) emission from PDS 70b with HST/WFC F336W filter and suggested that the hydrogen continuum emission dominates the UV flux. Aoyama et al. (2020) incorporated all these lines into a discussion of the emission mechanisms but were unable to determine fully the physical and accretion parameters of PDS $70 \mathrm{bc}$. 
Table 1

OSIRIS Observations using the Jn3 Filter with the Plate Scale of 20 mas

\begin{tabular}{lcc}
\hline \hline Target & $t_{\text {DIT }} \times n_{\text {DIT }}{ }^{\mathrm{a}}$ & $\begin{array}{c}\text { On-source Time } \\
(\mathrm{s})\end{array}$ \\
\hline PDS 70 & $40 \times 120$ & $4800^{(b)}$ \\
HD 143956 & $20 \times 1$ & 20 \\
HD 144609 & $2 \times 1$ & 2 \\
\hline
\end{tabular}

Note.

${ }^{\mathrm{a}} t_{\mathrm{DIT}}$ is the exposure time per image frame in the unit of seconds and $n_{\text {DIT }}$ is the number of image frames. ${ }^{b}$ The last eight frames were excluded in the analysis due to the inferior observing conditions, resulting in a practical total integration time of $3840 \mathrm{~s}$.

Here we report on a search for the previously unobserved line of $\mathrm{Pa} \beta(1.282 \mu \mathrm{m})$ around PDS 70 which is one of the brightest emission lines relative to $\mathrm{H} \alpha$. We used Keck/OSIRIS midresolution integral field spectroscopy (IFS; $R \sim 4000$ ) to further investigate the accretion mechanisms of PDS $70 \mathrm{bc}$. The observations and preliminary result of the postprocessing were originally reported in Uyama et al. (2021). In this paper we present the updated results with a detailed analysis of the data following Xie et al. (2020; see Sections 2 and 3). Section 4 investigates constraints on the accreting parameters of PDS $70 \mathrm{bc}$ by incorporating the OSIRIS results with the previous studies.

\section{Data \\ 2.1. Observations}

We observed PDS 70 with Keck/OSIRIS in the Jn3-band on 2020 May 31 UT (PI: Charles Beichman) to search for a $\mathrm{Pa} \beta$ emission line $(1.282 \mu \mathrm{m})$ from accretion onto the protoplanets. We used the OSIRIS IFS spatial sampling of 0 ". 02 spaxel $^{-1}$ that covers a field of view of 0 !" $96 \times 1$ !" 28 , where each spatial location has a spectrum from $1.275 \mu \mathrm{m}$ to $1.339 \mu \mathrm{m}$ (Jn3) with resolving power of $\sim 4000$. The observations achieved a total exposure time of $4800 \mathrm{~s}$ ( $120 \mathrm{~s}$ single exposure $\times 40$ frames) under good seeing conditions $(0$ ". 4-0" 6$)$. The typical full width half maximum (FWHM) of the PDS 70's point-spread function (PSF) measured a diffraction-limited $\sim 60-70$ mas, but the quality of the last sequence of the observations was poor because of high airmass $(>2.2)$ and relatively bad seeing $(\sim 0$ !"7). Hence we excluded the last eight frames from this analysis. By taking the ratio of the flux within a 3-by-3-spaxel aperture and within the entire field of view (FoV), we estimated the Strehl ratio to be $9.88 \%$ at $\mathrm{Pa} \beta$. Due to the relatively small FoV $(0$ ". $96 \times 1$ !' 28$)$, we may overestimate the Strehl ratio. The low Strehl ratio (typically $<20 \%$ ) can lead to flux loss and we took into account this effect in the data analysis. We also obtained unsaturated images of HD 143956 (spectral type: B9; Houk \& Smith-Moore 1988) and HD 144609 (spectral type: K0; Houk \& Smith-Moore 1988) for telluric correction and photometric reference, respectively. The details of the OSIRIS observations can be found in Table 5.

\subsection{Data Reduction}

We used the OSIRIS Data Reduction Pipeline (reduction type: astronomical reduction pipeline; Lyke et al. 2017; Lockhart et al. 2019) with the corresponding rectification matrices ${ }^{19}$ to extract

19 http://tkserver.keck.hawaii.edu/osiris/ the data cube and calibrated for dark subtraction, cosmic-ray removal, telluric correction, and wavelength solution. To search for faint companions with single emission lines, we need to first subtract the stellar light accurately. The preliminary data reduction presented in Uyama et al. (2021) applied the PCA-based SDI reduction that was originally used for the MUSE data (Hashimoto et al. 2020). However, this reduction technique left some instrumental residuals due to sensitivity differences between the OSIRIS spaxels. We therefore applied an advanced high-resolution spectral differential imaging (HRSDI) technique to remove the stellar emission (see Haffert et al. 2019 and Xie et al. 2020 for the details). HRSDI is suitable for retrieving sharp emission lines while removing the stellar halo. However, before we applied the HRSDI to the final combined data set, some residual bad pixels were removed from each exposure that passed through the OSIRIS Data Reduction Pipeline. To remove the bad pixels, we first applied HRSDI on each exposure, aiming for reducing the influence of stellar emission in the next step. Next, we applied a sigma clipping algorithm on the dithered exposures to make a bad pixel mask for each exposure. Then all the exposures were centered on the flux peak and mean combined after the removal of bad pixels.

The process of HRSDI consists of two steps, removing the stellar emission and removing the uncalibrated instrumental effects. The stellar emission was subtracted from all normalized spaxels with the normalized reference spectrum that was obtained after the continuum normalization (Haffert et al. 2019). The uncalibrated instrumental residuals were removed using a principal component analysis (PCA) subtraction technique (Amara \& Quanz 2012; Soummer et al. 2012). For example, the instrumental residual in Uyama et al. (2021) can be removed with the first few PCA components. The number of PCA components to subtract was determined by maximizing the signal-to-noise ratio of injected fake planets at the location of PDS $70 \mathrm{~b}$ (see also Section 2.3).

\subsection{Fake Planet Injection}

To estimate the instrumental throughput, we performed the fake planet injection described in Xie et al. (2020). The instrumental throughput includes the flux loss due to the low Strehl ratio (see Section 2.1) and that made by the PSF subtraction. Unless we specifically mentioned, both effects were corrected throughout the paper. The fake planet was created based on a planet spectrum and a stellar PSF. We used a single Gaussian line as the planet spectrum because our observations did not utilize angular differential imaging and thus did not achieve sufficient contrast to detect the continua of PDS $70 \mathrm{bc}$. We adopted the line-of-sight redshift of $25 \mathrm{~km} \mathrm{~s}^{-1}$ (Haffert et al. 2019) and a FWHM of $70 \mathrm{~km} \mathrm{~s}^{-1}$ or $0.3 \mathrm{~nm}$. The injected Gaussian line can be covered by two spectral channels. We measured the flux using the aperture photometry in spectral channels of $1281.75 \mathrm{~nm}$ and $1281.90 \mathrm{~nm}$ with a square aperture of 3-by-3 spaxels $(60 \times 60$ mas $)$. The noise was estimated at the same spatial location in the spectral direction after HRSDI, using 150 spectral channels (bandwidth: $22.5 \mathrm{~nm}$ ) around $\mathrm{Pa} \beta$. After obtaining a $5 \sigma$ detection, we estimated the flux loss caused by the PSF subtraction by comparing the injected and recovered flux. The flux losses caused by the PSF subtraction are $28 \%$ and $14 \%$ at the location of PDS $70 \mathrm{~b}$ and c, respectively. 


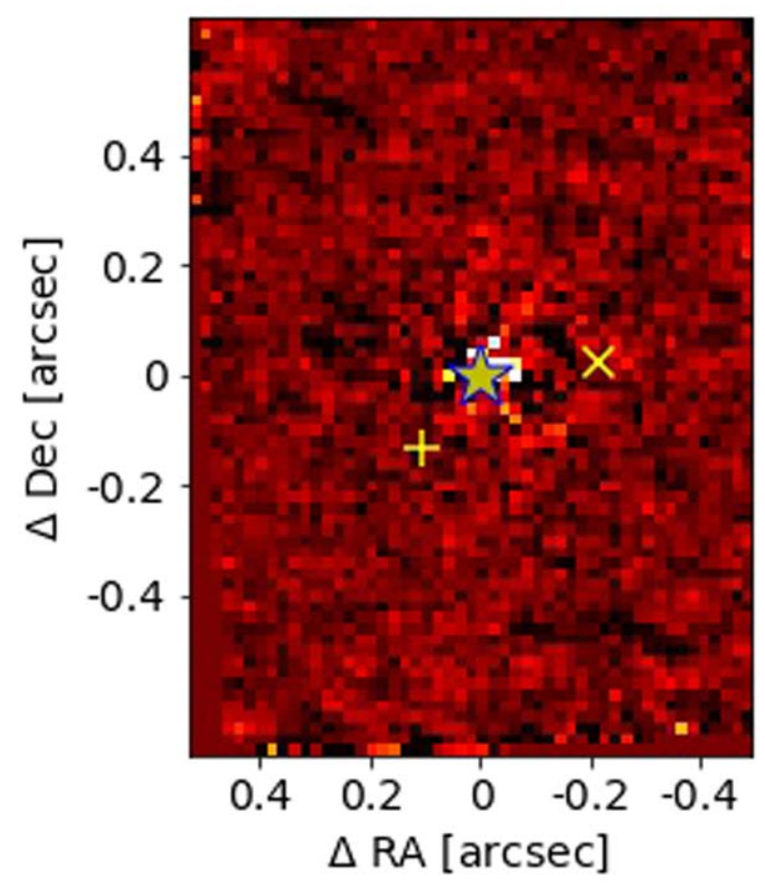

Figure 1. The HRSDI-reduced Keck/OSIRIS data at wavelengths of 1281.75 and $1281.9 \mathrm{~nm}$ (combined image). The locations of PDS 70, PDS $70 \mathrm{~b}$, and c are indicated by the star, plus, and cross symbols, respectively.

\section{Results}

After the postprocessing as mentioned in Section 2.2 we did not detect $\mathrm{Pa} \beta$ at the locations of PDS $70 \mathrm{~b}$ and $\mathrm{c}$ (see Figure 1). Figure 2 shows the residual spectra after the HRSDI reduction at the location of PDS 70bc. We then calculated the $5 \sigma$ detection limits ${ }^{20}$ of $1.4 \times 10^{-16} \mathrm{erg} \mathrm{s}^{-1} \mathrm{~cm}^{-2}$ and $1.9 \times 10^{-16}$ $\mathrm{erg} \mathrm{s}^{-1} \mathrm{~cm}^{-2}$ for PDS 70b and c, respectively. The correction of the flux loss caused by the PSF subtraction and the low Strehl ratio has been taken into account. Figure 3 shows the radial profiles for $5 \sigma$ detection limits at the two position angles of the two planets. We note that the PSF of OSIRIS is not circularly symmetric. Although PDS $70 \mathrm{c}$ is further away from the star, the noise at the location of PDS $70 \mathrm{c}$ is higher, resulting in a higher detection limit.

Uyama et al. (2021) defined the noise as a standard deviation of a spectral channel at the location of PDS $70 \mathrm{~b}$ after the SDI reduction without taking into account the OSIRIS' spectral resolution and flux loss by the postprocessing. Their calculations also used the literature value of PDS $70 \mathrm{~J}$-band flux $(J=9.553 \mathrm{mag}$; Skrutskie et al. 2006) to convert the contrast limit into a flux detection limit, but the central star is variable due to its activity and potentially also veiling by the circumstellar disk. In this study we used a field star of HD 144609 ( $J=5.459$ mag; Skrutskie et al. 2006) as a photometric reference and calculated a conversion factor from ADU to the apparent flux.

We also investigated the validity of the estimated limits by injecting fake sources. We used Aoyama \& Ikoma (2019) to convert the MUSE-based $\mathrm{H} \alpha$ profiles into the $\mathrm{Pa} \beta$ profiles

\footnotetext{
20 The $5 \sigma$ detection limit is defined as the summation of the flux in the aperture on the residual image and five times of the corresponding noise. As mentioned in Section 2.3, the estimated noise (without throughput correction) at the locations of PDS $70 \mathrm{~b}$ and $\mathrm{c}$ are $2.5 \times 10^{-18} \mathrm{erg} \mathrm{s}^{-1} \mathrm{~cm}^{-2}$ and $2.7 \times 10^{-18}$ erg s $\mathrm{cm}^{-1}$, respectively. The residual fluxes at the locations of PDS $70 \mathrm{~b}$ and $\mathrm{c}$ are $-2.7 \times 10^{-18} \mathrm{erg} \mathrm{s}^{-1} \mathrm{~cm}^{-2}$ and $2.5 \times 10^{-18} \mathrm{erg} \mathrm{s}^{-1} \mathrm{~cm}^{-2}$, respectively.
}

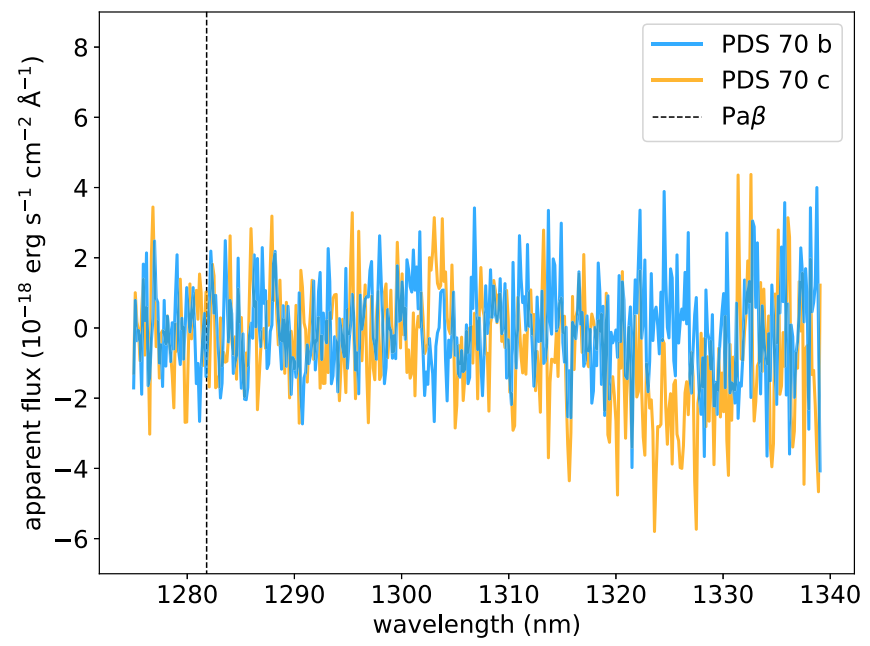

Figure 2. Spectra of the residuals after the HRSDI reduction at the locations of PDS $70 \mathrm{~b}$ and $\mathrm{c}$. For display purposes, no throughput correction was made.

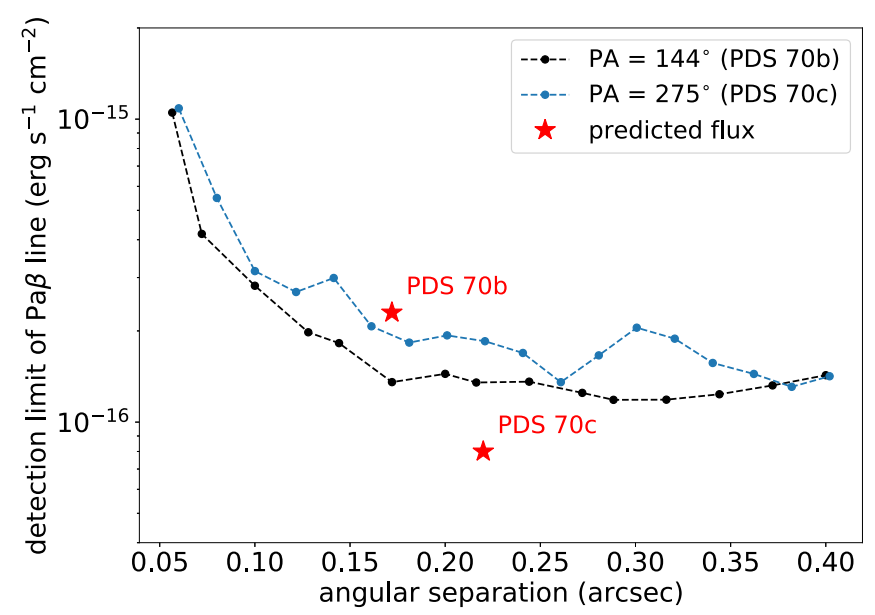

Figure 3. Radial profiles of $5 \sigma$ detection limits at two position angles. The predicted $\mathrm{Pa} \beta$ fluxes of PDS $70 \mathrm{bc}$ assuming the estimated parameters of Hashimoto et al. (2020) are indicated by the red stars.

assuming the derived parameters of PDS $70 \mathrm{bc}$ (the number density: $n_{0}=3.8 \times 10^{12} \mathrm{~cm}^{-3}$, the gas velocity: $v_{0}=144$ $\mathrm{km} \mathrm{s}^{-1}$, and the extinction: $A_{\mathrm{H}_{\alpha}}=2.4 \mathrm{mag}$ ) in Hashimoto et al. (2020). Our prediction for the $\mathrm{Pa} \beta$ flux from PDS $70 \mathrm{~b}$ is comparable to the actual OSIRIS detection limit. Since we did not detect $\mathrm{Pa} \beta$ emission our model may have overestimated the $\mathrm{Pa} \beta$ flux. Alternatively, Hashimoto et al. (2020) may have overestimated the $10 \%$ and $50 \%$ widths of the $\mathrm{H} \alpha$ profiles and thus the parameters of $n_{0}$ and/or $v_{0}$, possibly because MUSE does not have sufficient spectral resolution $(R \sim 2500)$. This latter interpretation can explain the difference between the mass measurements from the IR SED (e.g., Stolker et al. 2020; Wang et al. 2020) and the hydrogen-emission lines (Hashimoto et al. 2020). The mass estimate in Hashimoto et al. (2020) using the Aoyama \& Ikoma (2019) model is an upper limit on the dynamical mass of PDS $70 \mathrm{~b}$.

\section{Discussion}

We use our detection limits of $\operatorname{Pa} \beta$ to further constrain the physical parameters of PDS $70 \mathrm{bc}$ with a theoretical model (Aoyama et al. 2018; Aoyama \& Ikoma 2019). For a comparison 

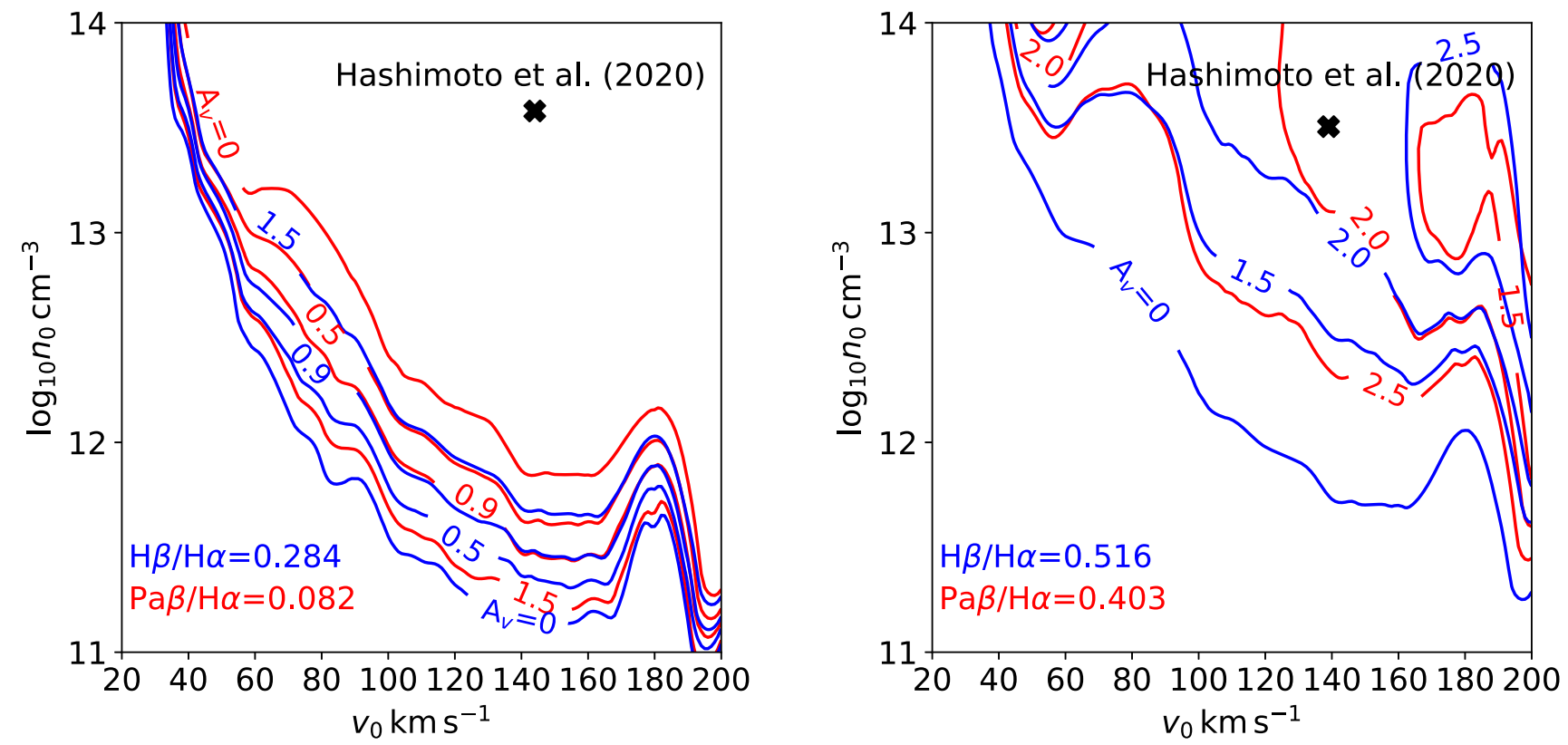

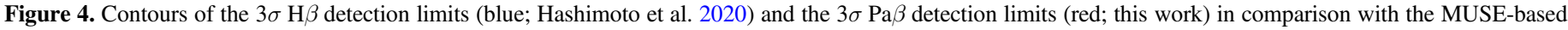

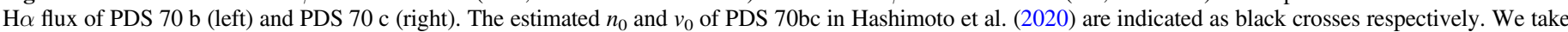

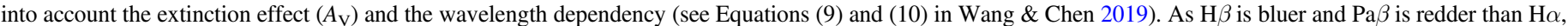
using these detection limits enables us to set upper and lower limits for $A_{\mathrm{V}}$, from which we estimate $A_{\mathrm{V}}$.

with our $\mathrm{Pa} \beta$ detection limits, we refer to: (1) the MUSE results (Hashimoto et al. 2020) which is most similar to the OSIRIS data rather than MagAO or HST because of the similarity of the data format and postprocessing techniques; and (2) the HST results (Zhou et al. 2021) that were obtained in 2020 May close to when we observed PDS 70 with OSIRIS, thereby mitigating any effects of the year-timescale intrinsic variability.

We assume magnetospheric accretion (filling factor of the hydrogen emission - the coverage fraction of the shock on the planetary surface: $\left.f_{\mathrm{f}} \lesssim 0.1\right)$ for the accretion mechanism of PDS 70 bc (e.g., Thanathibodee et al. 2019), from which we can set a lower limit in the $\left(n_{0}, v_{0}\right)$ parameter space (see also Figure 3 in Hashimoto et al. 2020 for the modeled $\mathrm{H} \alpha$ luminosity with different filling factor values). With this assumption, $\mathrm{H} \beta$ and $\mathrm{Pa} \beta$ line strengths are expected to be close to the MUSE and OSIRIS detection limits, respectively. Detailed explanations about the relationship between filling factor, other accretion parameters, and hydrogen-emission luminosity are given in Aoyama et al. (2020). If the filling factor is much larger than the above assumption we cannot simply compare the $\mathrm{Pa} \beta$ limits with the theoretical model. For example, when the shock comes from the circumplanetary disk surface flow rather than the magnetospheric accretion, the filling factor is a few tens of percent (Takasao et al. 2021).

\subsection{Comparison between the OSIRIS and MUSE Results}

Instrumental differences in the comparison of the visible and IR data should be small since the MUSE and OSIRIS IFSs have similar properties and the two data sets were treated in a similar fashion, using HRSDI to remove the stellar halo and searching for emission lines at small angular separations. We compare our $\mathrm{Pa} \beta$ detection limits with the MUSE-based $\mathrm{H} \alpha$ fluxes. However, we note that we have the uncertainty of time variability due to the difference of the epochs.

We used our $3 \sigma \mathrm{Pa} \beta$ detection limits $\left(6.6 \times 10^{-17} \mathrm{erg} \mathrm{s}^{-1} \mathrm{~cm}^{-2}\right.$ and $1.3 \times 10^{-16} \mathrm{erg} \mathrm{s}^{-1} \mathrm{~cm}^{-2}$ for PDS $70 \mathrm{~b}$ and $\mathrm{c}$, respectively) and the MUSE-based $\mathrm{H} \alpha$ fluxes and $3 \sigma \mathrm{H} \beta$ limits (Hashimoto et al. 2020) to constrain the PDS 70 bc's parameters. Combining the hydrogen-line data from these two AO-fed integral field units provides better constraints on the effects of extinction. The difference of extinction effect between $\mathrm{H} \beta / \mathrm{H} \alpha$ and $\mathrm{Pa} \beta / \mathrm{H} \alpha$ ratios enables us to estimate the $A_{\mathrm{V}}$ value. Figure 4 shows the contours of line flux ratio as a function of $n_{0}$ and $v_{0}$, with a variety of $A_{\mathrm{V}}$ values for PDS $70 \mathrm{~b}$ (left) and c (right), respectively. Although our final detection limit is higher than the preliminary result presented in Uyama et al. (2021), the comparison between the $\mathrm{Pa} \beta$ and $\mathrm{H} \beta$ limits suggests that $A_{\mathrm{V}}$ for the line emitting region of PDS $70 \mathrm{~b}$ is consistent with $\sim 0.9\left(A_{\mathrm{H} \alpha} \sim 0.69\right.$ mag assuming the extinction law in Wang \& Chen 2019).

Our extinction estimates are lower than other estimates. Hashimoto et al. (2020) attributed the failure of MUSE to detect $\mathrm{H} \beta$ to large extinction $\left(A_{\mathrm{H} \alpha}>2.0 \mathrm{mag}\right)$ but this may be due to the overestimation of $\left(n_{0}, v_{0}\right)$ and due to the insufficient spectral resolution of MUSE as mentioned in Section 3. Our derived $A_{\mathrm{V}}$ value is also inconsistent with the spectral energy distribution (SED)-fitting argument with the GRAVITY observations $\left(A_{\mathrm{V}} \sim 4-10 \mathrm{mag}\right.$ assuming ISM extinction and the best-fit extincted models; Wang et al. 2021) that used the shape of the continuum and the molecular-mapping argument from SINFONI observations $\left(A_{\mathrm{V}} \sim 16-17 \mathrm{mag}\right.$; Cugno et al. 2021), which used the depths of the lines. However, this discrepancy might suggest a vertical difference between the location of the photosphere responsible for the IR-continuum and the hydrogen-emitting regions. The evaporated materials at the shock can sublimate beneath the hydrogen-emitting regions to create an additional extinction source for the PDS $70 \mathrm{~b}$ 's atmosphere. This assumption does not conflict with the physical assumption of Aoyama et al. (2018). In that sense, IR-continuum observations and hydrogen-emission observations of protoplanets should be careful to identify each extinction effect independently. The large difference between $\left(n_{0}, v_{0}\right)$ estimated in Hashimoto et al. (2020), and the $\left(n_{0}, v_{0}\right)$ 
contour with $A_{\mathrm{V}}=0.9$ mag suggests that the filling factor may be larger than a lower limit of Hashimoto et al. (2020) $\left(f_{\mathrm{f}} \gtrsim 0.01\right)$ by about an order of magnitude. To test the hypothesis about the filling factor, observing the hydrogenemission line with higher spectral resolution is required. We note that the discussion in this section ignores the time variability as mentioned above. Section 4.2 takes into account the variability effect.

For the case of PDS $70 \mathrm{c}$, we could not explore as deep parameter space as PDS $70 \mathrm{~b}$ because the $\mathrm{Pa} \beta$ detection limit is higher than that of PDS $70 \mathrm{~b}$ as mentioned in Section 3 and the H $\alpha$ flux is smaller (Haffert et al. 2019; Hashimoto et al. 2020). The comparison with the $\mathrm{Pa} \beta$ and $\mathrm{H} \beta$ detection limits suggests $A_{\mathrm{V}} \sim 2.0 \mathrm{mag}\left(A_{\mathrm{H} \alpha} \sim 1.5 \mathrm{mag}\right)$. Compared with Hashimoto et al. (2020), who set a lower limit of $A_{\mathrm{H} \alpha}$ and $f_{\mathrm{f}}$ to $1.1 \mathrm{mag}$ and $\sim 0.003$, respectively, our estimated $A_{\mathrm{V}}$ value is consistent with their argument. We note that this argument relies on the assumption that the $\mathrm{H} \alpha$ profile of PDS $70 \mathrm{c}$ was sufficiently resolved by MUSE. If $n_{0}$ and $v_{0}$ of PDS $70 \mathrm{c}$ given in Hashimoto et al. (2020) are overestimated as well as those of PDS $70 \mathrm{~b}$, higher contrast levels at $\mathrm{H} \beta$ and $\mathrm{Pa} \beta$ are required to constrain these parameters. As mentioned above, PDS 70 c's photospheric continuum may also be extincted by additional material compared with the hydrogen-emitting regions. Resolving the $\mathrm{H} \alpha$ line profile with higher resolution and/or deeper searches for $\mathrm{H} \beta$ and $\mathrm{Pa} \beta$ will improve the constraints on the accretion parameters of PDS $70 \mathrm{c}$.

\subsection{Comparison between the OSIRIS and HST Results}

As mentioned above, the OSIRIS and MUSE observations were not conducted in the same epoch and thus simply comparing these observational results leaves the uncertainty of the temporal variability. Zhou et al. (2021) monitored PDS 70 b's $\mathrm{H} \alpha$ line with HST between 2020 February and 2020 July, which covers the OSIRIS observation on 2020 May 31 UT, and did not find larger variability in the line flux than $30 \%(\sim 2.4 \sigma)$. They also suggested the hydrogen-line emission was variable on a year timescale by incorporating MagAO and MUSE results obtained in 2018 (Wagner et al. 2018; Hashimoto et al. 2020). In this section we compare our $\mathrm{Pa} \beta$ detection limit of $\mathrm{PDS} 70 \mathrm{~b}$ with the timeaveraged $\mathrm{H} \alpha$ flux estimated from the HST observations $\left(1.62 \pm 0.23 \times 10^{-15} \mathrm{erg} \mathrm{s}^{-1} \mathrm{~cm}^{-2}\right.$; Zhou et al. 2021). Although the HST data format and postprocessing technique are different from OSIRIS, we used injection testing to account for differences in instrumental parameters and data analysis techniques. Figure 5 shows the same contours of PDS $70 \mathrm{~b}$ as Figure 4 assuming our $\mathrm{Pa} \beta$ limit and the HST-based $\mathrm{H} \alpha$ flux. Note that we do not include the $\mathrm{H} \beta$ limits because the $\mathrm{H} \beta$ observations were not conducted at the same epoch. The higher $\mathrm{H} \alpha$ flux value than the MUSE result helps us to explore a deeper parameter space. Assuming that the extinction effect is stable at $A_{\mathrm{V}}=0.9$ mag, our $3 \sigma$ detection limit can set an upper limit of $v_{0}$ at $\sim 70 \mathrm{~km} \mathrm{~s}^{-1}$. Using Equation (3) in Hashimoto et al. (2020), this upper limit corresponds to $\sim 3-4 M_{\text {Jup }}$ for the upper limit of PDS 70 b's mass and is consistent with the mass estimation by the IR high-contrast studies (e.g., Stolker et al. 2020; Wang et al. 2020). To better determine/constrain the (variable) accreting parameters simultaneous observations of $\mathrm{H} \alpha, \mathrm{H} \beta$, and $\mathrm{Pa} \beta$ are more helpful.

Zhou et al. (2021) estimated the continuum flux at the wavelength $\lambda=336 \mathrm{~nm}$ to be $(1.4 \pm 0.3) \times 10^{-18} \mathrm{erg} \mathrm{s}^{-1} \mathrm{~cm}^{-2}$ $\AA^{-1}$. This wavelength is located in the hydrogen Balmer continuum. Using the model of Aoyama et al. (2018), we can

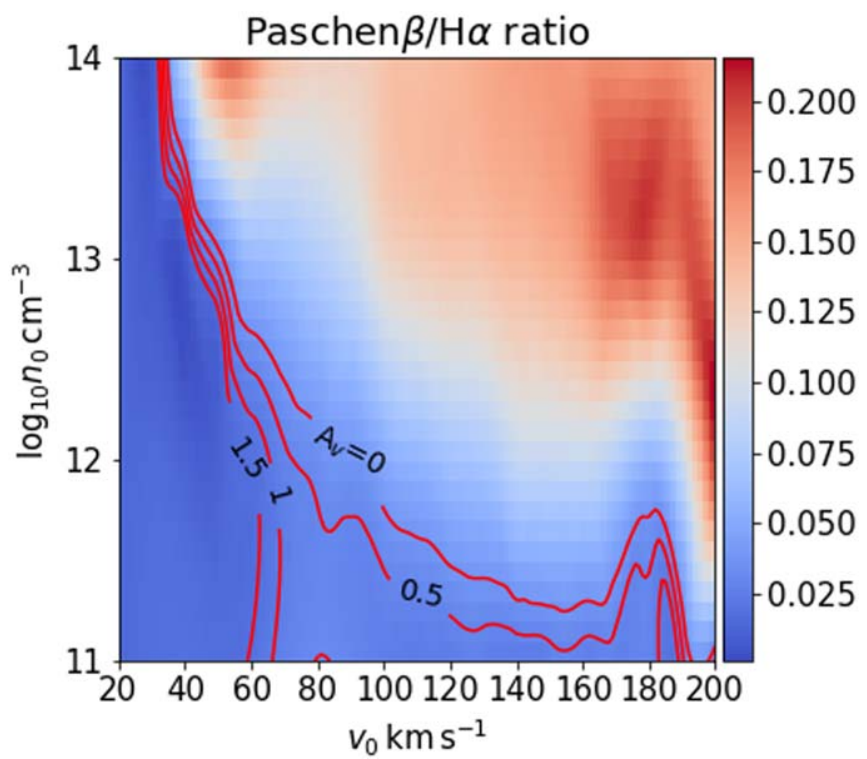

Figure 5. $3 \sigma \mathrm{Pa} \beta$ detection limit (red) in comparison with the HST-based $\mathrm{H} \alpha$ flux of PDS $70 \mathrm{~b}$. The contours assume $A_{\mathrm{V}}=0,0.5,1.0$, and 1.5 (from top to bottom) and the same wavelength dependency as Figure 4.

estimate the fluxes of the hydrogen recombination continua from the shock-heated gas, as a byproduct of the hydrogen-line fluxes. The model prediction can reproduce both the continuum and $\mathrm{H} \alpha$ fluxes observed for PDS $70 \mathrm{~b}$, with some parameter sets. However, our calculation with $\left(v_{0}, n_{0}\right)=\left(144 \mathrm{~km} \mathrm{~s}^{-1}, 3.8 \times 10^{12} \mathrm{~cm}^{-3}\right)$, which is estimated in Hashimoto et al. (2020), resulted in $F_{\lambda, 336} / F_{\mathrm{H} \alpha}=5.2 \times 10^{-3} \AA^{-1}$, where $F_{\lambda, 336}$ is the flux per unit wavelength at $\lambda=336 \mathrm{~nm}$ and $F_{\mathrm{H} \alpha}$ is the $\mathrm{H} \alpha$ flux, while its observed value is $(8.6 \pm 2.2) \times 10^{-4} \AA^{-1}$ when the flux in the F656N filter of HST represents the $\mathrm{H} \alpha$ flux (Zhou et al. 2021). This comparison shows inconsistency with the results of Hashimoto et al. (2020). This implies that the spectral profile given by MUSE can be overestimated, which is consistent with our interpretation about the null detection of $\mathrm{Pa} \beta$ in the OSIRIS observations. Note that the above estimate of $\left(v_{0}, n_{0}\right)$ comes from the MUSE-based spectral profile. However, the continuum flux for higher values of $v_{0}$ is less reliable due to a lack of coolants effective for hot gases in the Aoyama et al. (2018) model. Also, a part of photosphere that is heated by the accretion should emit continuum (e.g., Hartmann et al. 2016). Further theoretical studies on planetary recombination continua are essential.

\section{Summary}

We present high-contrast spectral imaging for the unexplored emission line of $\mathrm{Pa} \beta$ from $\operatorname{PDS} 70 \mathrm{bc}$ with Keck/ OSIRIS integral field spectroscopy. After removing stellar halo utilizing the same HRSDI technique that was applied to VLT/ MUSE observations, we did not detect $\mathrm{Pa} \beta$ despite the predicted $\mathrm{Pa} \beta$ flux of PDS $70 \mathrm{~b}$ from the estimated accretion parameters in Hashimoto et al. (2020) being comparable to the detection limit of our data set. The null detection suggests that our model overestimated the $\mathrm{Pa} \beta$ flux, probably because MUSE does not have sufficient spectral resolution and Hashimoto et al. (2020) overestimated $n_{0}$ and $v_{0}$ from the $\mathrm{H} \alpha$ profile.

We then compared our detection limits with previous $\mathrm{H} \alpha$ and $\mathrm{H} \beta$ observations to set further constraints on the accretion parameters. We adopted two $\mathrm{H} \alpha$ observations from MUSE and HST_comparing OSIRIS with MUSE can assume the smallest 
systematic difference in terms of the data format and postprocessing techniques, whereas HST covers 2020 May when we observed PDS 70 thereby minimizing the effect of time variability on our conclusions. The MUSE-based comparison between $\mathrm{Pa} \beta / \mathrm{H} \alpha$ and $\mathrm{H} \beta / \mathrm{H} \alpha$ ratios enables us to estimate $A_{\mathrm{V}}$ assuming the extinction law. We estimated $A_{\mathrm{V}} \sim 0.9$ and 2.0 for PDS $70 \mathrm{bc}$, respectively. Particularly the derived $A_{\mathrm{V}}$ of PDS $70 \mathrm{~b}$ is inconsistent with the previous NIR studies, but this might suggest an additional extinction source of PDS $70 \mathrm{~b}$ 's IR-continuum photosphere that is located beneath the hydrogen-emitting regions. The HST-based $\mathrm{Pa} \beta /$ $\mathrm{H} \alpha$ ratio suggested that the year-timescale variation does not significantly affect the $A_{\mathrm{V}}$ estimate. We also incorporated the Balmar continuum detected by HST/WFC F336W observations in the Aoyama et al. (2018) framework. The comparison between the Balmer continuum with $\mathrm{H} \alpha$ flux suggests that the $\mathrm{H} \alpha$ spectral profile may be overestimated. This interpretation is consistent with the null detection of $\mathrm{Pa} \beta$ in our OSIRIS observations.

Higher spectral resolution will resolve the hydrogenemission line profiles and a deeper search could detect multiple hydrogen emissions, which helps to better estimate the accreting parameters and understand the accretion mechanisms of protoplanets.

We thank Jim Lyke for kindly helping to confirm the OSIRIS FoV settings. The authors would like to thank the anonymous referees for their constructive comments and suggestions to improve the quality of the paper. T.U. is supported by Grant-inAid for Japan Society for the Promotion of Science (JSPS) Fellows and JSPS KAKENHI grant No. JP21J01220. Y.H. is supported by the Jet Propulsion Laboratory, California Institute of Technology, under a contract with NASA. K.W. acknowledges support from NASA through the NASA Hubble Fellowship grant HST-HF2-51472.001-A awarded by the Space Telescope Science Institute, which is operated by the Association of Universities for Research in Astronomy, Incorporated, under NASA contract NAS5-26555. The results reported herein benefited from collaborations and/or information exchange within NASA's Nexus for Exoplanet System Science (NExSS) research coordination network sponsored by NASA's Science Mission Directorate.

The data presented herein were obtained at the W. M. Keck Observatory, which is operated as a scientific partnership among the California Institute of Technology, the University of California and the National Aeronautics and Space Administration. The Observatory was made possible by the generous financial support of the W. M. Keck Foundation. This publication makes use of data products from the Two Micron All Sky Survey, which is a joint project of the University of Massachusetts and the Infrared Processing and Analysis Center/California Institute of Technology, funded by the National Aeronautics and Space Administration and the National Science Foundation.

We wish to acknowledge the critical importance of the current and recent Maunakea Observatories daycrew, technicians, telescope operators, computer support, and office staff employees, especially during the challenging times presented by the COVID-19 pandemic. Their expertize, ingenuity, and dedication is indispensable to the continued successful operation of these observatories. The authors wish to recognize and acknowledge the very significant cultural role and reverence that the summit of Maunakea has always had within the indigenous Hawaiian community. We are most fortunate to have the opportunity to conduct observations from this mountain.

\section{ORCID iDs}

Taichi Uyama (D) https://orcid.org/0000-0002-6879-3030 Chen Xie (1) https://orcid.org/0000-0002-6318-0104 Yuhiko Aoyama (i) https://orcid.org/0000-0003-0568-9225 Charles A. Beichman (10) https://orcid.org/0000-00025627-5471

Jun Hashimoto (iD https://orcid.org/0000-0002-3053-3575 Ruobing Dong (D) https://orcid.org/0000-0001-9290-7846 Yasuhiro Hasegawa (iD https://orcid.org/0000-0002-9017-3663 Masahiro Ikoma (iD https://orcid.org/0000-0002-5658-5971 Dimitri Mawet (1) https://orcid.org/0000-0002-8895-4735 Michael W. McElwain (i) https://orcid.org/0000-00030241-8956

Jean-Baptiste Ruffio (iD https://orcid.org/0000-00032233-4821

Kevin R. Wagner (iD https://orcid.org/0000-0002-4309-6343

Jason J. Wang (iD https://orcid.org/0000-0003-0774-6502

Yifan Zhou (i) https://orcid.org/0000-0003-2969-6040

\section{References}

Amara, A., \& Quanz, S. P. 2012, MNRAS, 427, 948

Aoyama, Y., \& Ikoma, M. 2019, ApJL, 885, L29

Aoyama, Y., Ikoma, M., \& Tanigawa, T. 2018, ApJ, 866, 84

Aoyama, Y., Marleau, G.-D., Mordasini, C., \& Ikoma, M. 2020, arXiv:2011. 06608

Bonnefoy, M., Currie, T., Marleau, G. D., et al. 2014, A\&A, 562, A111

Boss, A. P. 1997, Sci, 276, 1836

Christiaens, V., Cantalloube, F., Casassus, S., et al. 2019, ApJL, 877, L33

Cugno, G., Patapis, P., Stolker, T., et al. 2021, A\&A, 653, A12

Haffert, S. Y., Bohn, A. J., de Boer, J., et al. 2019, NatAs, 3, 749

Hartmann, L., Herczeg, G., \& Calvet, N. 2016, ARA\&A, 54, 135

Hashimoto, J., Aoyama, Y., Konishi, M., et al. 2020, AJ, 159, 222

Houk, N., \& Smith-Moore, M. 1988, Michigan Catalogue of Two-dimensional Spectral Types for the HD Stars, Vol. 4 (Ann Arbor, MI: Univ. of Michigan)

Keppler, M., Benisty, M., Müller, A., et al. 2018, A\&A, 617, A44

Lockhart, K. E., Do, T., Larkin, J. E., et al. 2019, AJ, 157, 75

Lyke, J., Do, T., Boehle, A., et al. 2017, OSIRIS Toolbox: OH-Suppressing InfraRed Imaging Spectrograph pipeline, Astrophysics Source Code Library, ascl:1710.021

Pollack, J. B., Hubickyj, O., Bodenheimer, P., et al. 1996, Icar, 124, 62

Skrutskie, M. F., Cutri, R. M., Stiening, R., et al. 2006, AJ, 131, 1163

Soummer, R., Pueyo, L., \& Larkin, J. 2012, ApJL, 755, L28

Spiegel, D. S., \& Burrows, A. 2012, ApJ, 745, 174

Stolker, T., Marleau, G. D., Cugno, G., et al. 2020, A\&A, 644, A13

Takasao, S., Aoyama, Y., \& Ikoma, M. 2021, arXiv:2106.16113

Thanathibodee, T., Calvet, N., Bae, J., Muzerolle, J., \& Hernández, R. F. 2019, ApJ, 885, 94

Uyama, T., Hashimoto, J., Beichman, C. A., et al. 2021, RNAAS, 5, 9

Wagner, K., Follete, K. B., Close, L. M., et al. 2018, ApJL, 863, L8

Wang, J. J., Ginzburg, S., Ren, B., et al. 2020, AJ, 159, 263

Wang, J. J., Vigan, A., Lacour, S., et al. 2021, AJ, 161, 148

Wang, S., \& Chen, X. 2019, ApJ, 877, 116

Xie, C., Haffert, S. Y., de Boer, J., et al. 2020, A\&A, 644, A149

Zhou, Y., Bowler, B. P., Wagner, K. R., et al. 2021, AJ, 161, 244 